\title{
EXPERIMENTAL ANALYSIS OF THE ACASIAS ACTIVE LINING PANEL EMUS 2020
}

\author{
S. ALGERMISSEN* AND M. MISOL* \\ * German Aerospace Center (DLR) \\ Institute of Composite Structures and Adaptive Systems \\ Braunschweig, Germany \\ e-mail: stephan.algermissen@dlr.de, web page: http://www.dlr.de
}

Key words: acoustic control, active lining, modal testing, thermal testing

\begin{abstract}
The participants of the EU-project ACASIAS develop advanced concepts for aerostructures with multifunctional capabilities. Within work package 3 an active system for noise reduction is integrated into a curved lining panel. The objective is the reduction of the interior sound pressure level by increasing the transmission loss of the lining panel. The application scope includes current propeller driven aircraft and future aircraft with counter-rotating open rotor (CROR) engines. The drawback of these $\mathrm{CO}_{2}$-efficient engines is their high sound emission in the frequency band up to $500 \mathrm{~Hz}$. Active noise reduction systems are able to achieve performance in this frequency band while passive sound insulation materials fall behind. Thus, active systems are the key technology to avoid unacceptably high noise levels for passengers.

During the last period of the project the main experiments are conducted. The active lining with its dimensions of $1300 \times 1690 \mathrm{~mm}^{2}(\mathrm{~W} \times \mathrm{H})$ is mounted in a setup in the acoustic transmission loss test facility of the DLR. Several tests in different categories are run to characterize the vibration and sound transmission behavior of the lining. In this paper the active lining and its components are briefly introduced and the results of the modal and the thermal testing are presented.
\end{abstract}

\section{INTRODUCTION}

The last half year marks the beginning of the experimental phase of ACASIAS work package (WP) 3. In the period before concepts have been developed to take active system for noise reduction in aircraft to the next level. Laboratory equipment is substituted by off-the-shelf components. These components are encapsulated to create a production-friendly integration approach [1]. System maintenance and repair solutions are provided. Control system algorithms are fine tuned to achieve the expected goals [2]. The following sections provide a summary of two selected experiments which are conducted with the ACASIAS active lining in the acoustic transmission loss test facility of DLR. After introducing the active lining, the results of the first experiment, the modal testing, are presented. Accompanying the construction of the lining a detailed Finite Element (FE) model has been setup by the WP 3 partner CIMNE. The objective of this experiment is to provide modal data within a certain bandwidth in order to update the 


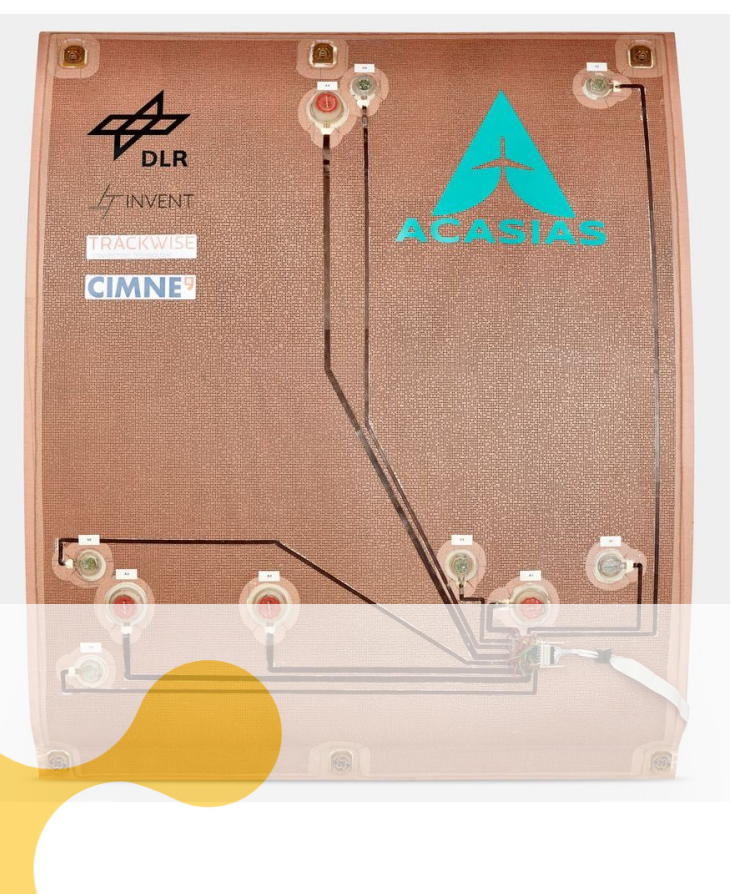

Figure 1: Active lining, backside

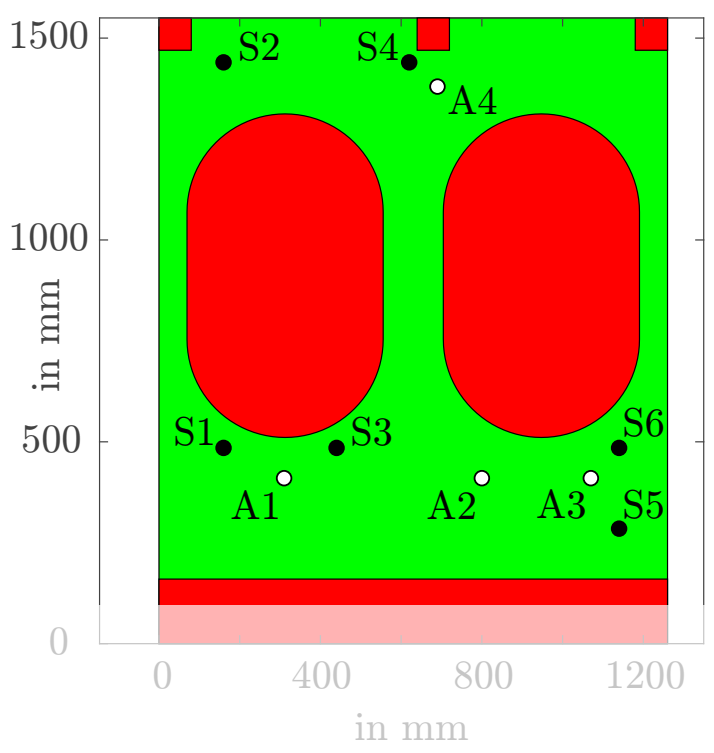

$\begin{array}{ll}- \text { Actuator } & \text { Forbidden area } \\ \text { - Sensor } & \text { Routing area }\end{array}$

Figure 2: Actuator (A\#) and sensor (S\#) placement,

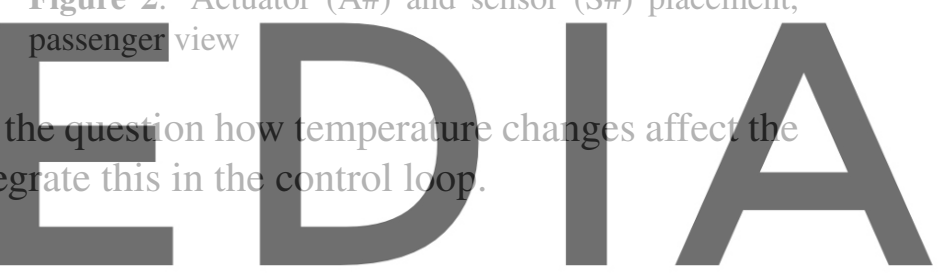

2 ACTIVE LINING

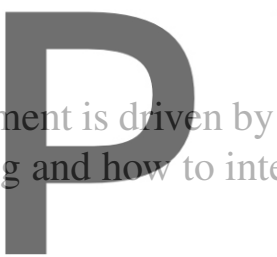

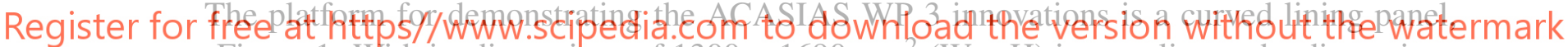 see Figure 1. With its dimensions of $1300 \times 1690 \mathrm{~mm}^{2}(\mathrm{~W} \times \mathrm{H})$ it complies to the dimensions of original A350 sidewall panels. The partners agreed to neglect the window cut-outs since} the effort and the costs for manufacturing an adequate mold would have exceeded the financial framework. Furthermore, the new technologies can be presented without loss of generality with the given approach.

The lining is designed as a sandwich part. An inner honeycomb core is stabilized by a top layer of glass fiber prepreg on each side. In series production the core is crushed in a press during the curing cycle. Since a press is not available for manufacturing at the partner's site, the active lining is manufactured with an intact honeycomb core. Nevertheless, the final thickness shall comply to the original one. The layup of the lining has been defined together with the industrial advisory board member DiEHL AVIATION. The inner layer is a fine meshed prepreg. It generates a very flat surface that faces the passengers. In series production the surface is equipped with customer-specific coating. On the inner layer a honeycomb core is placed. On top of the core a single layer of very rough and open glass fiber prepreg finalizes the layup. The target thickness of the final lining is approximately $7 \mathrm{~mm}$. At six positions, three at the upper and three at the lower edge, the lining is connected to the fuselage structure using shock mounts. Figure 3 shows the situation in the acoustic lab. For testing the lining is mounted in a 


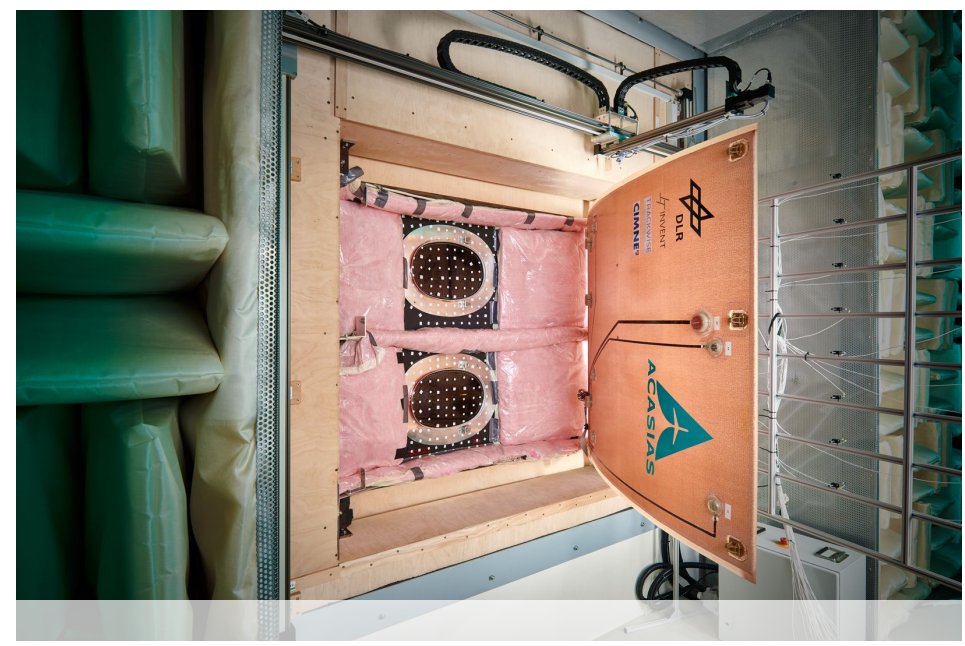

Figure 3: Lining in acoustic lab

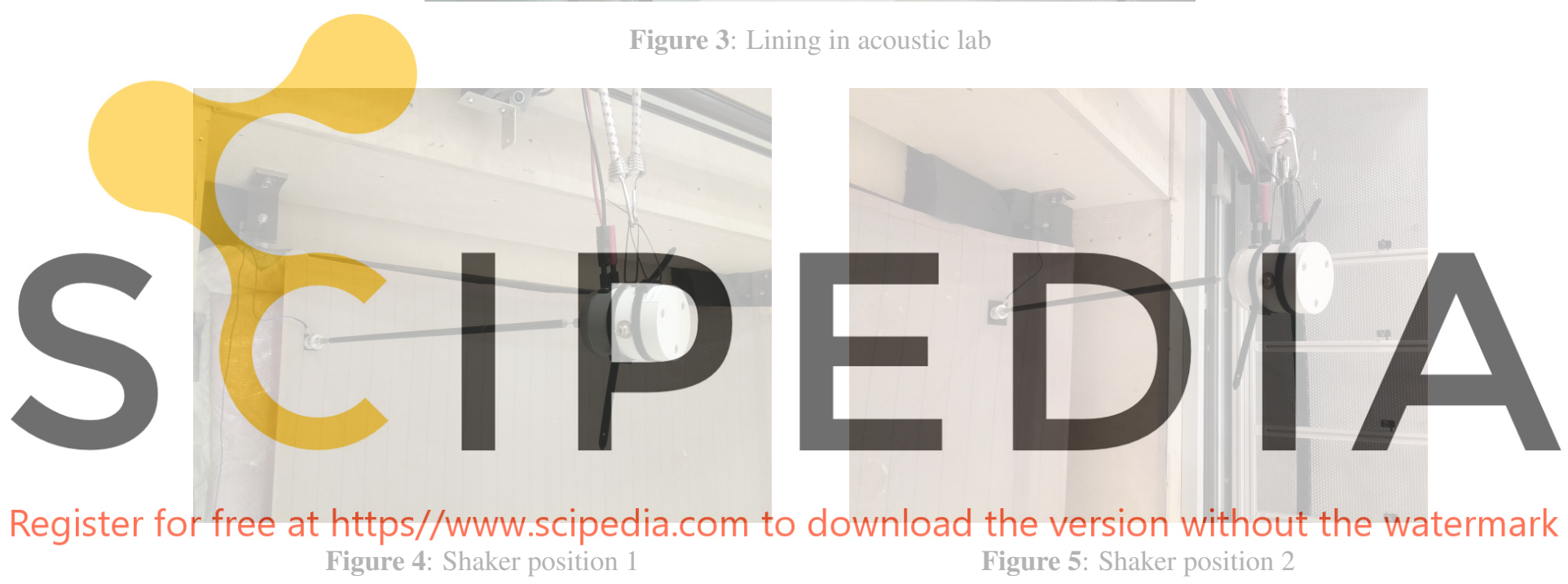

$90^{\circ}$ counterclockwise rotated position in front of an A350-like fuselage panel.

The lining panel is made "active" by including sensors, actuators and wiring in order to control its vibrations and therefore its sound radiation into the cabin. The sensors of the lining are accelerometers measuring the normal acceleration at the point of installation. The actuators introduce defined forces into the lining structure. In this project electrodynamic exciters are used instead of piezo actuators to achieve the desired performance at frequencies below $200 \mathrm{~Hz}$.

In a detailed analysis [2] the effect of the number of sensors and actuators on the control performance is investigated. The result leads to six sensors and four actuators which are placed on the lining according to the given criterion. Figure 2 summarizes the final placement of the components. For a transfer of results to a lining from series production, the areas with windows, air condition ducts and mounting points are treated as forbidden (red) with regard to placement and routing. The type of actuators and sensors and their integration technology is described in [1] in detail. 


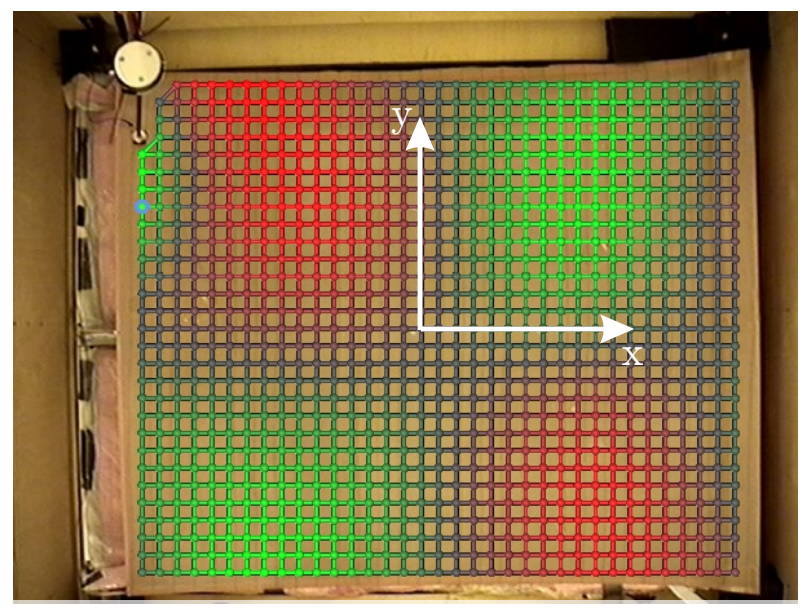

Figure 6: LSV scan point grid with coordinate system

\section{MODAL ANALYSIS}

An experimental modal analysis is conducted with the lining panel. The objective is to provide poles and mode shapes for the update of the FE model created within the project. The analysis is divided into two parts. First, the experimental data have to be obtained. Second,

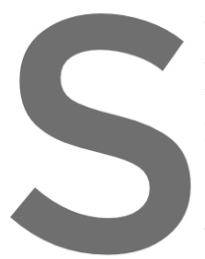
these data have to be merged and Beginning with the description in the following sections.

3.1 Setup
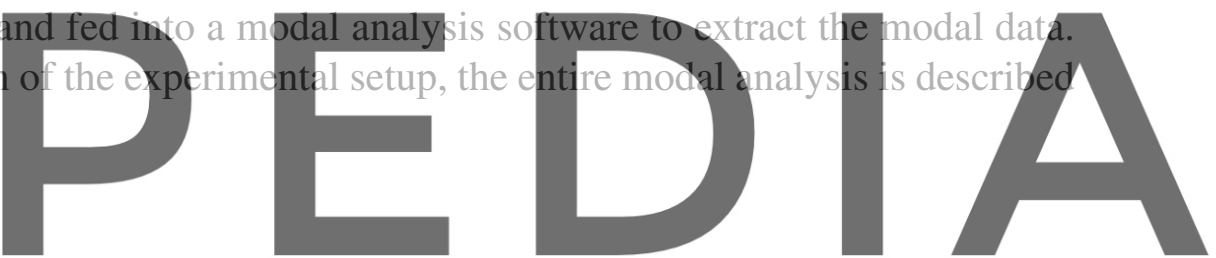

Nearly all technical structures vibrate and exhibit modal behavior. In order to describe it,

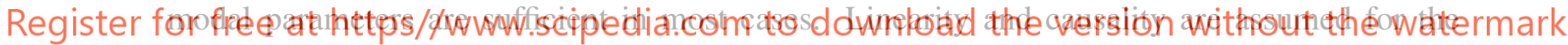
vibration behavior of the time-invariant structure under test. The input data for the modal analysis are frequency response functions (FRF) from force to displacement measured on a set of selected points of the given structure. Since the inner side of the lining which directs to the passengers is essential for describing the sound emission, a grid of approximately 1,000 points is specified on this surface. Each point has six degrees of freedom (DOF), three translational and three rotational. The relevant DOF for this application is the normal displacement of each surface point. The measurement of displacement at a huge amount of points is challenging. Therefore, a POLYTEC ${ }^{\circledR}$ PSV-400 laser scanning vibrometer (LSV) is used to determine the FRF from force input to point velocities. After calculation of the FRF the input unit is changed to displacement by division with $j \omega$.

The input force is generated by a shaker mounted hanging in front of the lining. The force is measured with a sensor of type PCB ${ }^{\circledR} 208 \mathrm{~B} 01$ which is bonded on the lining. To achieve modal completeness two different excitation points are selected, see Figure 4 and 5. The points are located at the upper edge of the lining panel with respect to the mounting direction. Position 1 is located $165 \mathrm{~mm}$ from the upper and $55 \mathrm{~mm}$ from the left edge. Whereas position 2 is $165 \mathrm{~mm}$ apart from the upper and the right edge. 


\begin{tabular}{ccc|ccc}
$\begin{array}{c}\text { Mode } \\
\text { number }\end{array}$ & $\begin{array}{c}\text { Undamped eigen- } \\
\text { frequency in Hz }\end{array}$ & $\begin{array}{c}\text { Damping } \\
\text { ratio in } \%\end{array}$ & $\begin{array}{c}\text { Mode } \\
\text { number }\end{array}$ & $\begin{array}{c}\text { Undamped eigen- } \\
\text { frequency in } \mathrm{Hz}\end{array}$ & $\begin{array}{c}\text { Damping } \\
\text { ratio in \% }\end{array}$ \\
\hline 1 & 27.2 & 2.8 & 8 & 98.3 & 2.6 \\
2 & 39.8 & 8.5 & 9 & 110.4 & 1.8 \\
3 & 44.9 & 4.1 & 10 & 127.6 & 3.5 \\
4 & 46.5 & 5.1 & 11 & 152.1 & 4.7 \\
5 & 59.5 & 2.3 & 12 & 159.1 & 3.4 \\
6 & 67.6 & 3.4 & 13 & 176.8 & 2.9 \\
7 & 77.8 & 3.1 & 14 & 197.7 & 2.7 \\
\hline
\end{tabular}

Table 1: Modal data

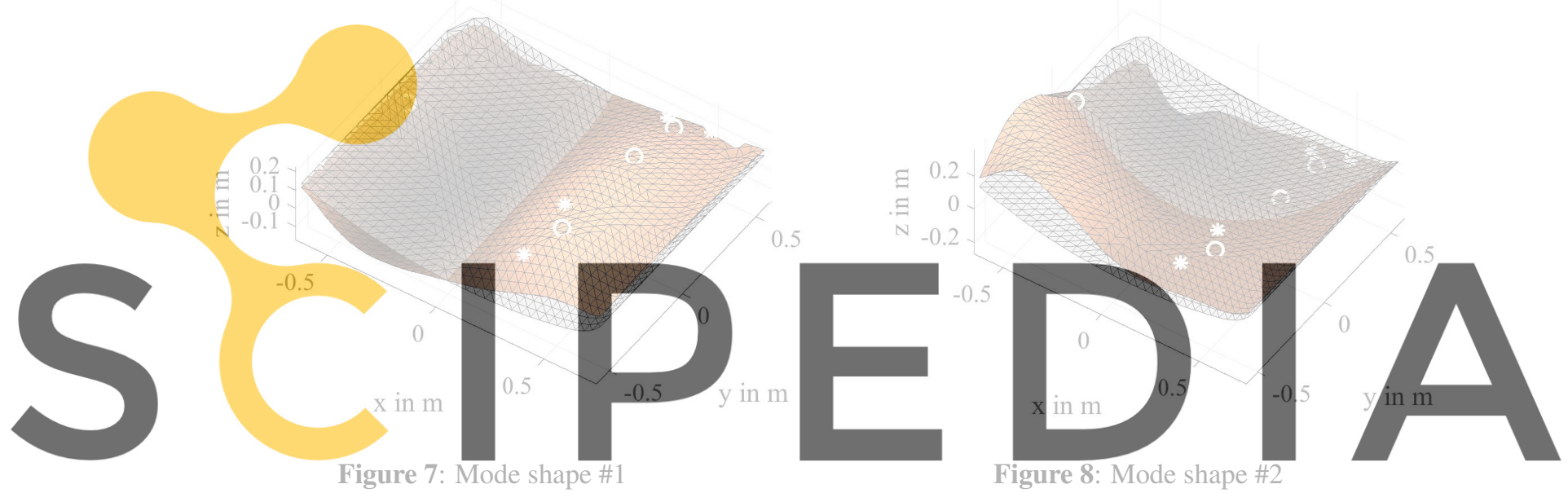

For testing the shaker is driven with a periodic chirp signal from the LSV. The bandwidth for the measurement is set to $1 \mathrm{kHz}$ while 1,600 FFT lines are used which leads to a frequency resolution of $0.625 \mathrm{~Hz}$ in the FRF. A three times complex averaging enhances the signal quality. The LSV scan point grid is shown in Figure 6. It is an even rectangular grid with 35 points in $x$ and 29 points in $y$-direction with a spacing of app. $40 \mathrm{~mm}$. Five points at the upper left corner are neglected due to their lack of visibility. Therefore, the final grid consists of 1,010 points.

Two measurements are conducted where the shaker is mounted in the positions defined above. The resulting FRF are merged with the modal analysis software XMODAL3. By means of the complex mode indicator function (CMIF) 14 relevant modes are identified in the bandwidth from 20 to $200 \mathrm{~Hz}$. The modal data are summarized in Table 1. The damping ratio lies in the interval from 1.8 to $8.5 \%$. All identified mode shapes are real-valued and orthogonal to each other. Figures 7-10 show shapes of selected modes. The circles denote the positions of the actuators while the asterisk marks the sensors. It becomes obvious that not all modes are symmetric. This is mainly due to the distribution of actuator and sensor masses. 


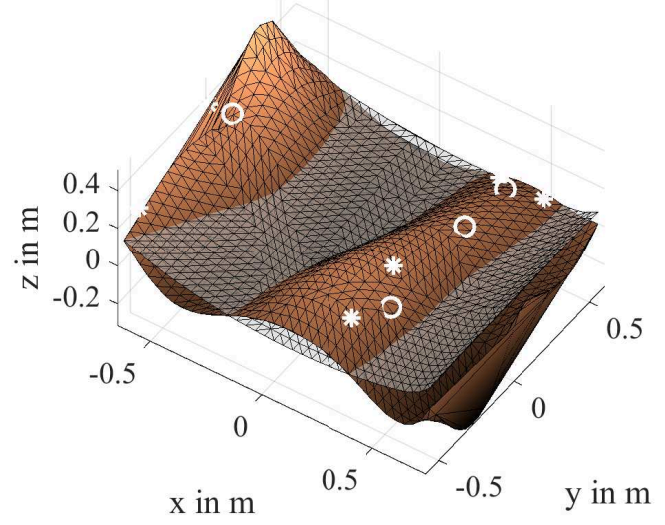

Figure 9: Mode shape \#6

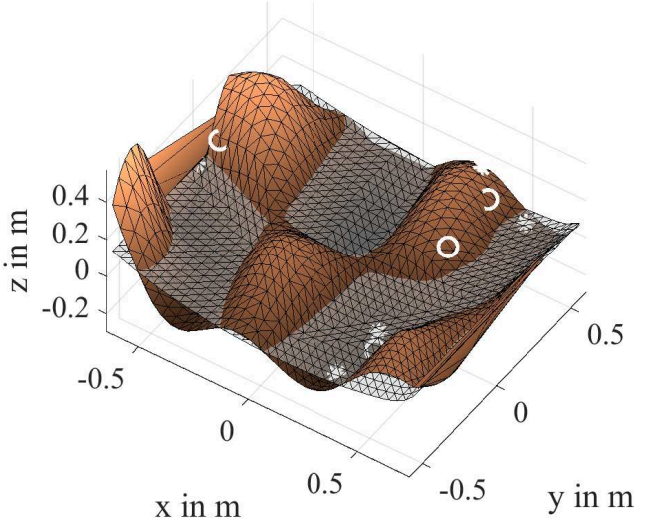

Figure 10: Mode shape \#10

\section{THERMAL EXPERIMENTS}

Lining panels in aircraft are subject to environmental influences such as humidity or temperature in the cabin. Although the climate in a cabin is controlled, the temperature of the lining panels may differ from the air condition set point temperature due to solar radiation for exam-

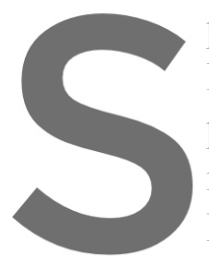
ple. Since the material parameters are a function of temperature, the vibration behavior of the lining panel changes with increasing temperatures as well. The active yolse reduction system presented in [3] must maintain its performance regardless of the temperature. Therefore, the influence of the temperature on the ribration behavior of the lining needs to be deternfined. In control, the accuracy of the secondary path model $\underline{G}$ is elementary for the stability of the entire control system. The objective of the experiments presented in the following section is the

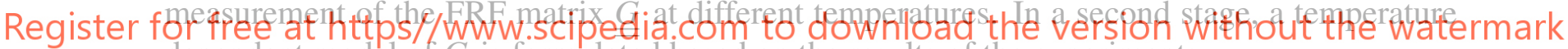
dependent model of $\underline{G}$ is formulated based on the results of the experiments.

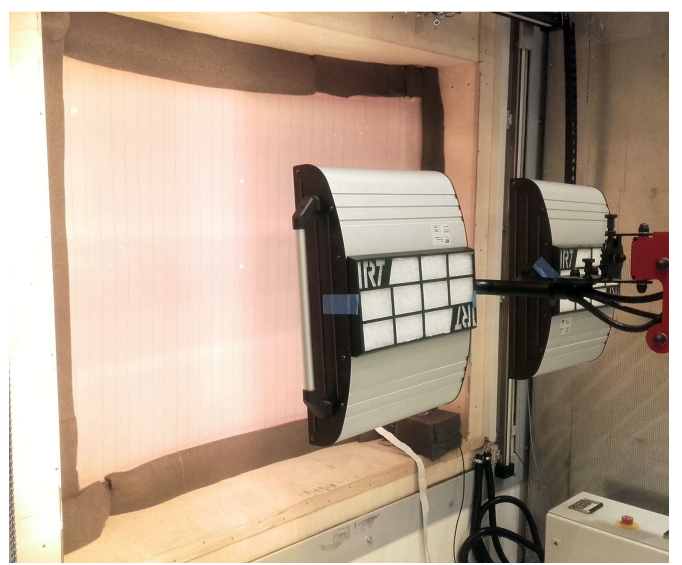

Figure 11: IR dryer in front of lining panel

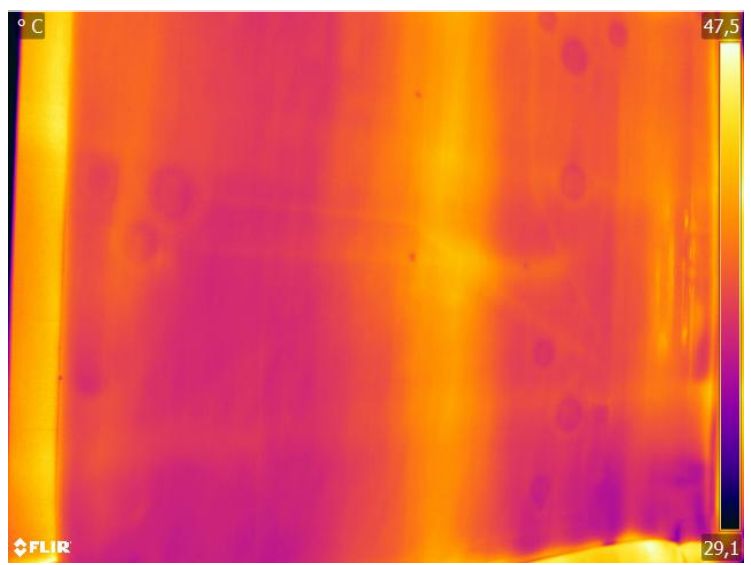

Figure 12: Thermal camera image at set point $35^{\circ} \mathrm{C}$ 


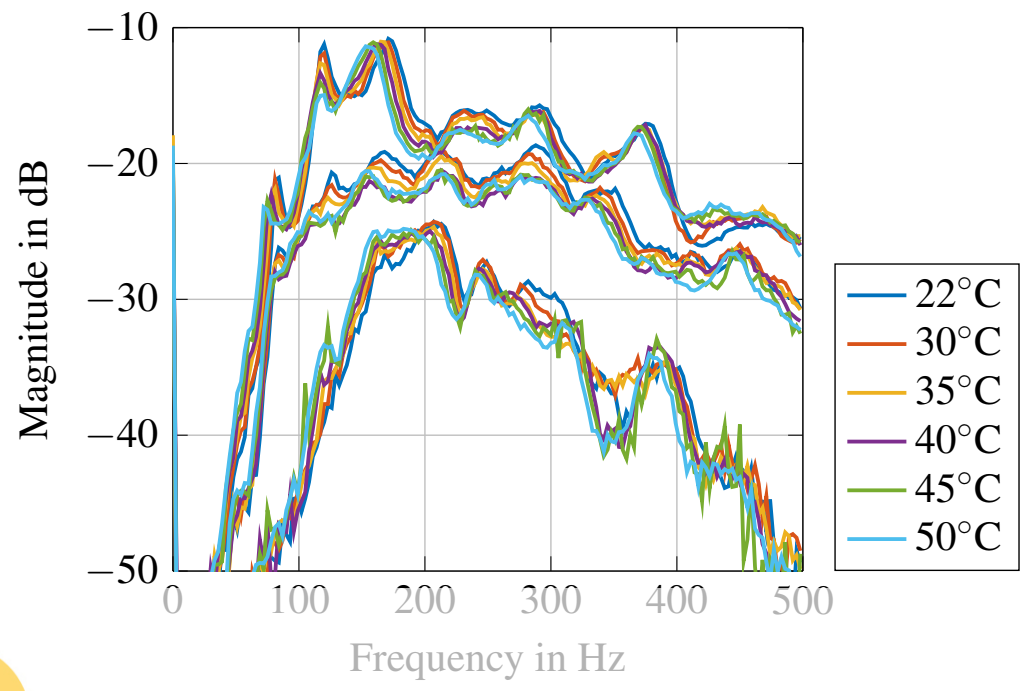

Figure 13: Singular values of $\underline{G}$ at temperatures $T_{S}$

\subsection{Setup}

The temperature ranges for airborne equipment are defined in the EUROCAE ED-14G [4]

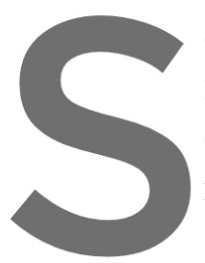

chapter four. Lining pa

from $-15^{\circ} \mathrm{C}$ to $+55^{\circ} \mathrm{C}$

equipped with a cooling

range from ambient 22

The thermal experim
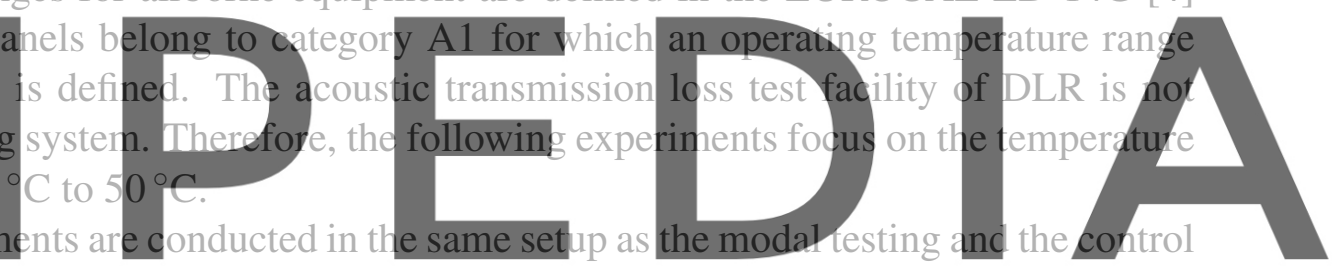

experiments. The even heating of the lining's surface is realized with an infrared (IR) dryer of

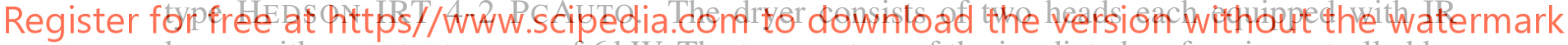

lamps with an output power of $6 \mathrm{~kW}$. The temperature of the irradiated surface is controlled by

the dryer based on contact-free temperature measurements. To monitor the heating process, an additional temperature probe is mounted on the backside of the lining close to the lower left corner. A thermal imaging camera of type FLIR ${ }^{\circledR}$ A615 is used to observe the temperature distribution on the surface of the lining. Figure 11 shows the setup in the lab.

The secondary control path consists of the rapid prototyping control system, the actuators and the sensors of the lining. Additional anti-aliasing and reconstruction filters are placed in the signal paths. The overview of the entire control loop is given in [3]. The number of sensors $n_{y}=6$ and actuators $n_{u}=4$ define the dimensions of $\underline{\underline{G}} 6 \times 4$.

As set points for the experiments the following temperatures from ambient to $50{ }^{\circ} \mathrm{C}$ are defined:

$$
T_{s}=\left[\begin{array}{llllll}
22 & 30 & 35 & 40 & 45 & 50
\end{array}\right] \text { in }{ }^{\circ} \mathrm{C} .
$$

As temperature reference the probe on the backside is used. The set point of the IR dryer controller is adjusted according to the probe. A steady state with respect to the lining temperature is achieved by a 11 min time span of the heating process at each set point. The temperature distribution is checked with the thermal imaging camera at each set point. Figure 12 gives an impression of the distribution at $35^{\circ} \mathrm{C}$. The view axis of the camera is equivalent to the one in 

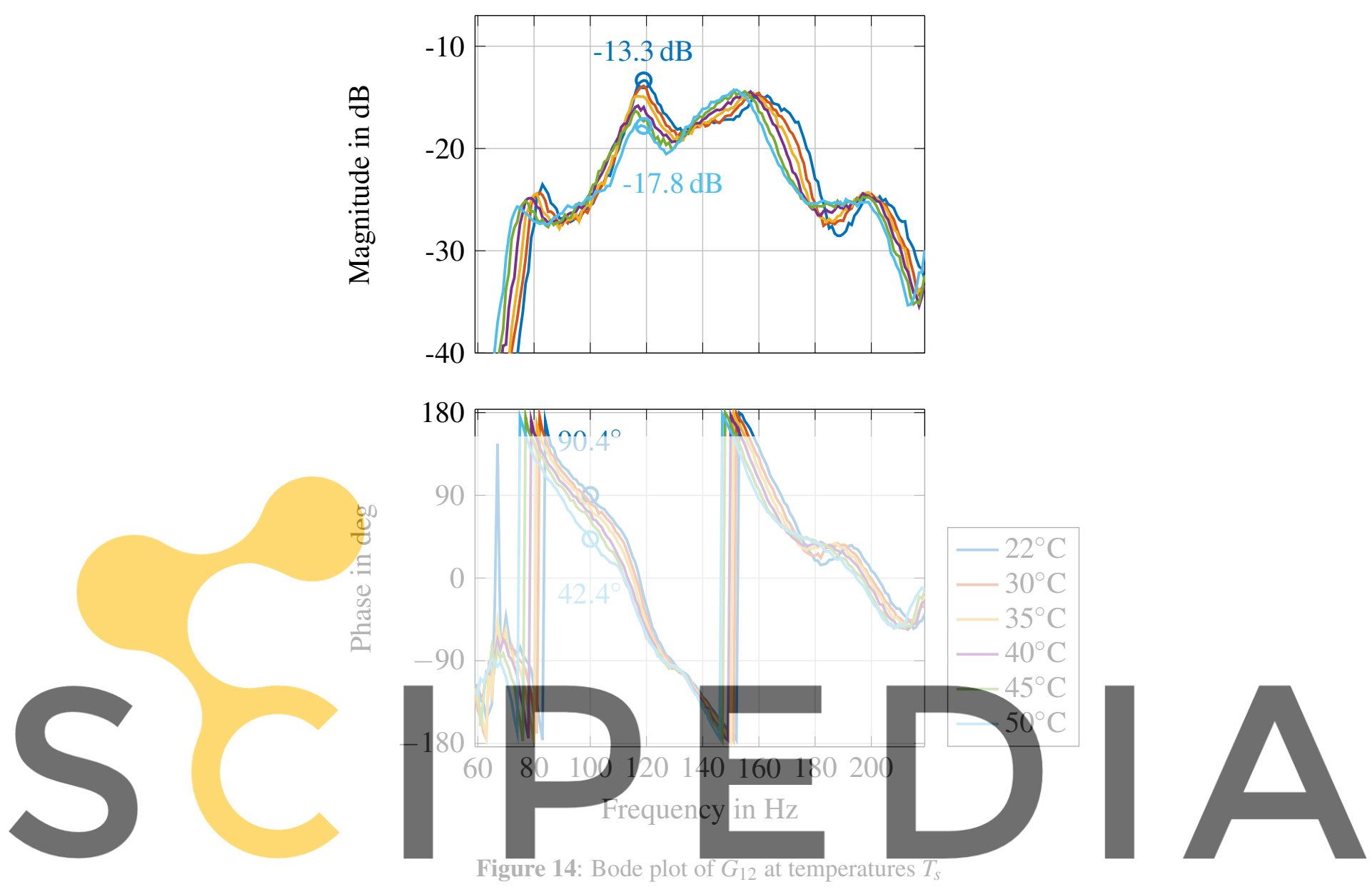

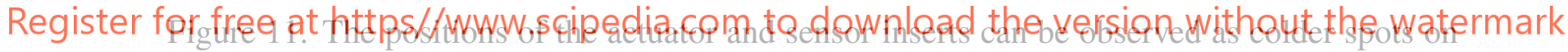
the lining.

\subsection{Results}

The identification of the secondary path $\underline{G}$ is conducted with the rapid prototyping system. The four actuators are driven with uncorrelated noise signals for $64 \mathrm{~s}$ while the six sensors record the lining's responses. The bandwidth of the identification is set to $1000 \mathrm{~Hz}$ since the cut-off frequency of the anti-aliasing and reconstruction low-pass filters are set to $500 \mathrm{~Hz}$. The FRF of $\underline{G}$ is calculated with 64 times averaging which results in a frequency resolution of $1 \mathrm{~Hz}$. During the experiment actuator 3 and sensor 6 had a failure at certain temperatures. Therefore, the two signal channels are neglected in the subsequent analysis.

To compare the FRF at temperatures $T_{s}$, the singular values of $\underline{\underline{G}}$ are shown in Figure 13 in the relevant bandwidth. It becomes obvious that $\underline{\underline{G}}$ is temperature dependent and that in general the eigenfrequencies decrease with increasing temperature. The shift of eigenfrequencies is moderate and smooth. A stepwise change is not observed. In a first, simplified assumption the shift can be explained with a decreasing stiffness of the lining towards higher temperatures.

To quantify the shift, the Bode plot $G_{12}$ from actuator 2 to sensor 1 is considered more closely in Figure 14. In this example the magnitude difference in the eigenfrequency at $119 \mathrm{~Hz}$ is 


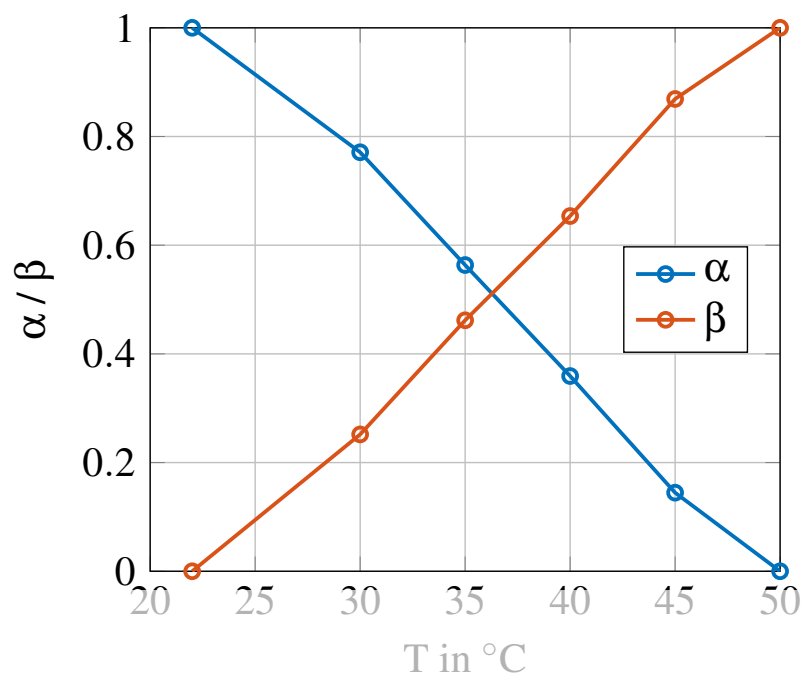

Figure 15: Factors $\alpha$ and $\beta$ as a function of temperature T

$4.5 \mathrm{~dB}$. Due to the shifting eigenfrequency around $80 \mathrm{~Hz}$ the phase difference at $100 \mathrm{~Hz}$ amounts to $48^{\circ}$ while the magnitude remains constant. Magnitude and phase errors in this order may lead to instability of the control loop. These results confirm the assumption that a temperature dependent secondary path model $\underline{G}(T)$ is needed for operation of the system in aircraft.

As few measurements as necessary should be accomplished to generate $\underline{\underline{G}}(\boldsymbol{T})$ shifts of the eigenfrequencies
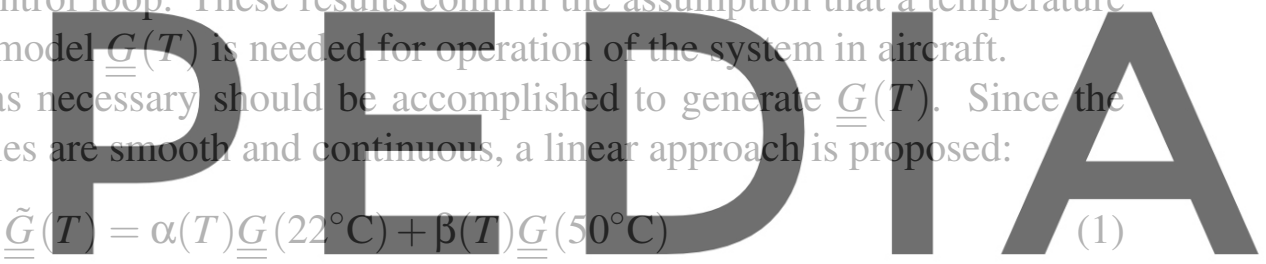

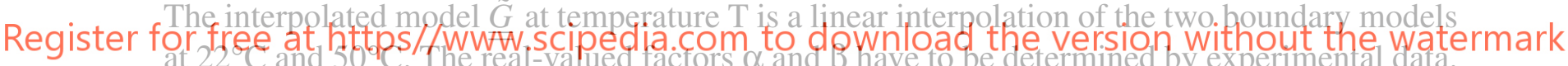

Therefore, (1) is rearranged to:

$$
\underline{\underline{\tilde{G}}}(T)=\left[\underline{\underline{G}}\left(22^{\circ} \mathrm{C}\right) \underline{\underline{G}}\left(50^{\circ} \mathrm{C}\right)\right]\left[\begin{array}{l}
\alpha(T) \\
\beta(T)
\end{array}\right]
$$

Since the FRF are also matrices, functions of frequency $\omega$ and complex-valued, Equation (2) expands to:

$$
\underbrace{\left[\begin{array}{c}
\mathfrak{R}\left(G_{11}\left(\omega_{1}, T\right)\right) \\
\vdots \\
\mathfrak{R}\left(G_{n_{y} n_{u}}\left(\omega_{1}, T\right)\right) \\
\mathfrak{I}\left(G_{11}\left(\omega_{1}, T\right)\right) \\
\vdots \\
\mathfrak{I}\left(G_{n_{y} n_{u}}\left(\omega_{1}, T\right)\right) \\
\vdots \\
\mathfrak{I}\left(G_{n_{y} n_{u}}\left(\omega_{N}, T\right)\right)
\end{array}\right]}_{\underline{\underline{A}}} \underbrace{\left[\begin{array}{cc}
\mathfrak{R}\left(G_{11}\left(\omega_{1}, 22^{\circ} \mathrm{C}\right)\right) & \mathfrak{R}\left(G_{11}\left(\omega_{1}, 50^{\circ} \mathrm{C}\right)\right) \\
\vdots & \vdots \\
\mathfrak{R}\left(G_{n_{y} n_{u}}\left(\omega_{1}, 22^{\circ} \mathrm{C}\right)\right) & \mathfrak{R}\left(G_{n_{y} n_{u}}\left(\omega_{1}, 50^{\circ} \mathrm{C}\right)\right) \\
\mathfrak{I}\left(G_{11}\left(\omega_{1}, 22^{\circ} \mathrm{C}\right)\right) & \mathfrak{I}\left(G_{11}\left(\omega_{1}, 50^{\circ} \mathrm{C}\right)\right) \\
\vdots & \vdots \\
\mathfrak{I}\left(G_{n_{y} n_{u}}\left(\omega_{1}, 22^{\circ} \mathrm{C}\right)\right) & \mathfrak{I}\left(G_{n_{y} n_{u}}\left(\omega_{1}, 50^{\circ} \mathrm{C}\right)\right) \\
\vdots & \vdots \\
\mathfrak{I}\left(G_{n_{y} n_{u}}\left(\omega_{N}, 22^{\circ} \mathrm{C}\right)\right) & \mathfrak{I}\left(G_{n_{y} n_{u}}\left(\omega_{N}, 50^{\circ} \mathrm{C}\right)\right)
\end{array}\right]}_{\underline{\underline{B}}}\left[\begin{array}{c}
\alpha(T) \\
\beta(T)
\end{array}\right]
$$




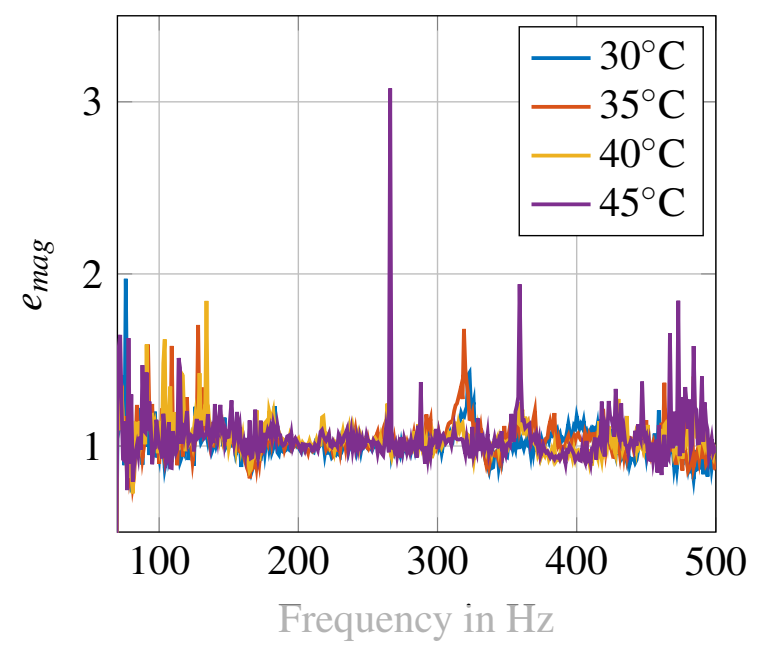

Figure 16: Magnitude error

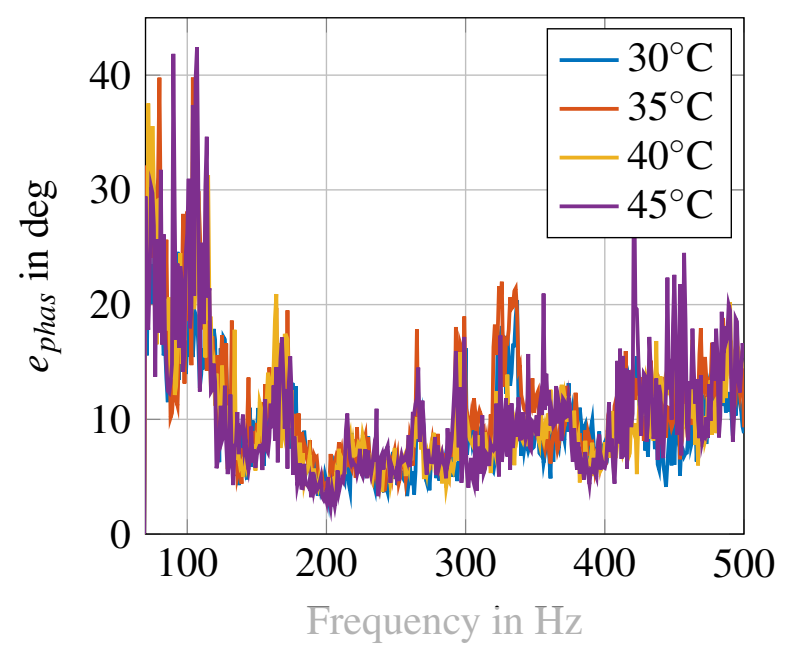

Figure 17: Phase error

Where $\Re()$ and $\mathfrak{J}()$ denote the real and the imaginary part and $N$ the number of frequencies. On the left hand side $\underline{\tilde{G}}(T)$ is substituted by the measured $\underline{G}(T)$ in order to solve the equation system for $\alpha$ and $\beta$ at each temperature $T_{s}$. The solution is found by building the Moore-Penrose pseudoinverse ()$^{+}$:
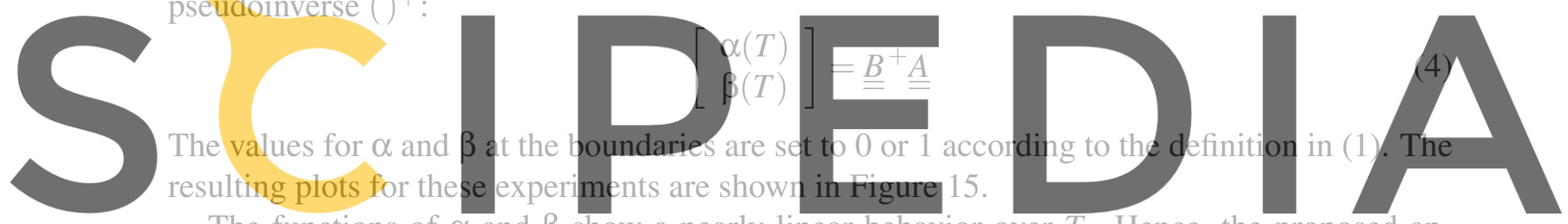

The functions of $\alpha$ and $\beta$ show a nearly linear behavior over $T$. Hence, the proposed apRegister for ared in (1) together with a linear formulatign of the factors seems to be suitable for a first approach to model the temperature dependent behavior of the $G$. To validate the benefit of the interpolated model $\tilde{G}$ at temperatures $T_{S}$, the mean magnitude and phase errors are calculated.

Therefore, all single FRF in the transfer matrix of $\underline{\underline{\underline{G}}}(\omega, T)$ are divided by the corresponding measured $\underline{\underline{G}}(\omega, T)$ at temperature $T$. The frequency dependent mean magnitude and phase errors are calculated by summation:

$$
\begin{aligned}
e_{\text {mag }}\left(\omega_{n}, T\right) & =\frac{1}{n_{u} n_{y}} \sum_{u=1}^{n u} \sum_{y=1}^{n y}\left|\frac{\tilde{G}_{y u}\left(\omega_{n}, T\right)}{G_{y u}\left(\omega_{n}, T\right)}\right| \\
e_{\text {phas }}\left(\omega_{n}, T\right) & =\frac{1}{n_{u} n_{y}} \sum_{u=1}^{n u} \sum_{y=1}^{n y}\left|\angle\left(\frac{\tilde{G}_{y u}\left(\omega_{n}, T\right)}{G_{y u}\left(\omega_{n}, T\right)}\right)\right|
\end{aligned}
$$

Applied to the experimental data, the plots in Figures 16 and 17 result. Despite some peaks, the values for $e_{m a g}$ are closely located to the desired value of 1 . The phase error $e_{\text {phas }}$ exceeds $20^{\circ}$ below $120 \mathrm{~Hz}$ while it remains under $20^{\circ}$ for nearly all frequencies up to $500 \mathrm{~Hz}$. To put the errors into a context, a second error calculation is performed. It is considered the case that only the base model $\underline{\underline{G}}\left(22^{\circ} \mathrm{C}\right)$ exists. The lining is heated up to $40^{\circ} \mathrm{C}$ and the secondary path 


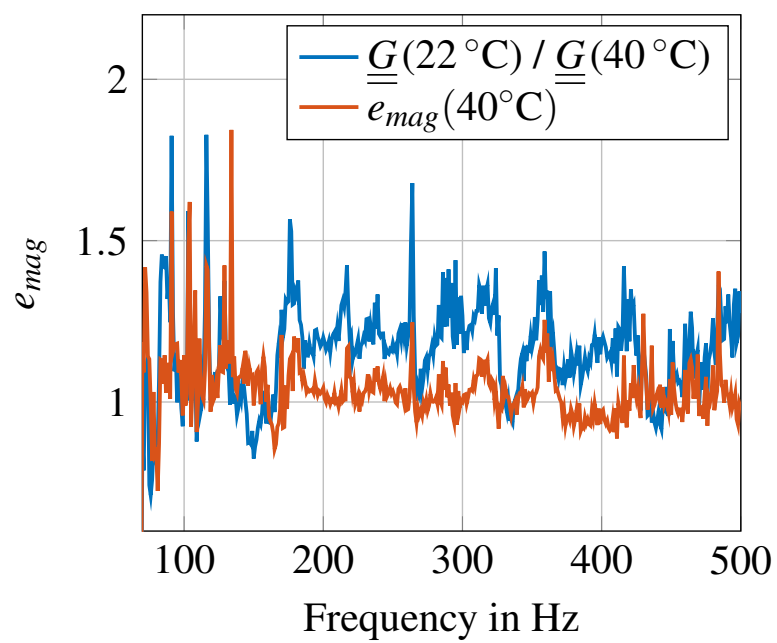

Figure 18: Magnitude error

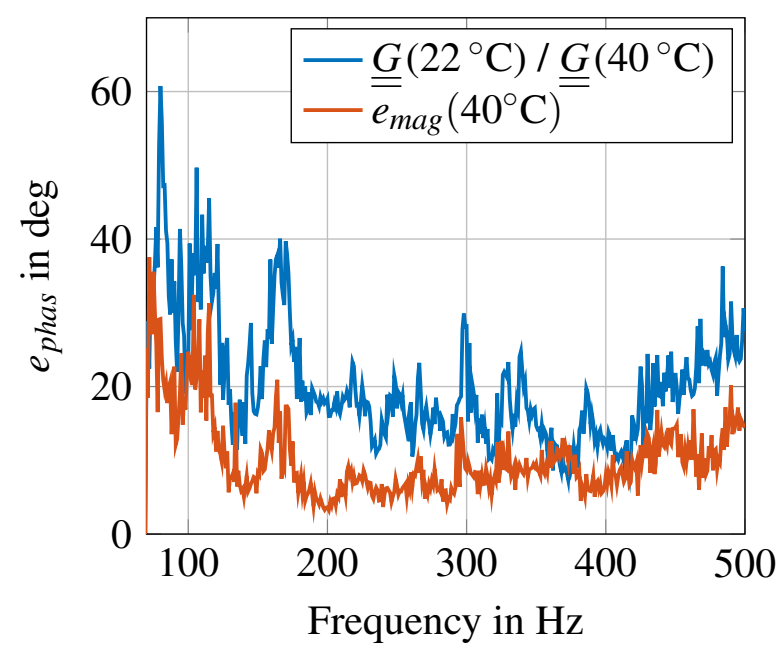

Figure 19: Phase error

model behaves like $\underline{G}\left(40^{\circ} \mathrm{C}\right)$. The error that occurs is calculated with (5) and (6) while $\underline{\tilde{G}}$ is substituted by $\underline{\underline{G}}\left(22^{\circ} \mathrm{C}\right)$. Figure 18 and 19 compare this error to $e_{m a g}\left(40^{\circ} \mathrm{C}\right)$ and $e_{\text {phas }}\left(40^{\circ}{ }^{\overline{\bar{O}}}\right)$.

It is obvious that the interpolated model $\underline{\underline{\tilde{G}}}$ significantly reduces the error in nearly all relevant frequencies in the control bandwidth. Thus, the use of the interpolated model instead of the exclusive use of the base model $\underline{\underline{G}}\left(22^{\circ} \mathrm{C}\right)$ is preferred. To set up the interpolated model, only a single extra measurement at $50{ }^{\circ} \overline{\overline{\mathrm{C}}}$ has to be conducted in this case.

\section{CONCLUSIONS \& OUTLOOK}

The realizations and the results of two main experiments of the last phase of the ACASIAS WP 3 are presented. Within the modal analysis 14 modes in the bandwidth from 20 to $200 \mathrm{~Hz}$ could be determined. Real modes and realistic damping ratios are identified. The modal data now serve as a base for the model updating procedure of the lining's FE model. Beyond that, the effects of different ambient temperatures on the secondary path of the control loop are quantified in a second experiment. It has been shown that the effects cannot be neglected when the noise reduction system becomes operational. An approach is proposed to describe the temperature dependency by a linear combination of two boundary models. This approach enables a simple tracking of the varying vibration behavior in order to adjust the controller and to optimize its performance over a wider temperature span. Future work will concentrate on the implementation of these results in the controller software.

\section{ACKNOWLEDGMENTS}

This project has received funding from the European Union's Horizon 2020 research and innovation programme under grant agreement No. 723167.

The authors gratefully acknowledge the support of the industrial advisory board member DieHl AviATion, Laupheim, Germany. 


\section{REFERENCES}

[1] S. Algermissen, M. Misol, A. Kokott, T. Haase, K. Gonet, and V. Lungaho, "Towards a lining integrated active structural acoustic control system," in Proc. of European Conference on Multifunctional Structures (EMuS), X. Martinez and H. Schippers, Eds., Juni 2019, pp. 30-37. [Online]. Available: https://elib.dlr.de/127884/

[2] M. Misol and S. Algermissen, "Remote sensing for a lining integrated structural acoustic control system," in Proc. of European Conference on Multifunctional Structures (EMuS), X. Martinez and H. Schippers, Eds., Barcelona, Spain, 2019. [Online]. Available: https://elib.dlr.de/128067/

[3] — - "Noise reduction results of the ACASIAS active lining panel," in Proc. of European Conference on Multifunctional Structures (EMuS), X. Martinez and H. Schippers, Eds., 2020.

[4] European Organisation for Civil Aviation Equipment (EUROCAE), EUROCAE ED-14G: Environmental Conditions and Test Procedures for Airborne Equipment. EUROCAE, 2011. 